\title{
Dynein regulates cell migration depending on substrate rigidity
}

\author{
DAEHWAN KIM, EUNAE YOU and SANGMYUNG RHEE \\ School of Biological Sciences (BK21 Program), Chung-Ang University, Seoul 156-756, Republic of Korea
}

Received November 1, 2011; Accepted December 8, 2011

DOI: $10.3892 / \mathrm{ijmm} .2011 .867$

\begin{abstract}
The mechanical environment in combination with biochemical signaling is an important regulatory factor for cellular physiology including tissue development, cell motility and differentiation. Exerting a tractional force triggered by myosin-dependent cell contractility is known to be an indispensible element of cell migration in a mechanically stiff environment such as a 2D planar surface. However, a number of reports have argued that the requirement of myosin activity for cell migration is limited by cell type and the environment. In this study, we present evidence that dynein, a minus enddirected microtubule motor, plays a central role in cell migration in the absence of tractional force. Interfering with the dynein activity through a dynein-specific inhibitor, erythro-9-(2hydroxy-3-nonyl) adenine (EHNA), dramatically inhibited 2D migration of the fibroblast when cell contractility was blocked by Rho kinase or a myosin inhibitor, although EHNA itself did not affect cell migration. Cell migration in 3D soft collagen matrices, where the cell exerts a relatively low tractional force compared to that on a 2D stiff surface, is also profoundly inhibited by dynein intermediate chain (DIC) silencing regardless of the presence of myosin activity. In addition, DIC-silenced cells on a soft acrylamide surface show decreased migration without blockade of myosin activity. Taken together, our results suggest that dynein may be a primary regulatory factor for cell migration when a cell is in a mechanically low-tension environment, such as in a $3 \mathrm{D}$ matrix.
\end{abstract}

\section{Introduction}

The mechanical and physical features of tissues organized by the extracellular matrix (ECM) are known to be important for biological processes including embryo development, cell migration and tissue differentiation (1). It has been clearly shown that the mechanical and physical features of the ECM environment affect cell morphology. For example, cell culture on a rigid culture dish or glass coverslip produces a highly

Correspondence to: Dr Sangmyung Rhee, School of Biological Sciences (BK21 Program), Chung-Ang University, Seoul 156-756, Republic of Korea

E-mail: sangmyung.rhee@cau.ac.kr

Key words: cell migration, tractional force, dynein, myosin, microtubule, 3D matrix flattened morphology with stress fibers. On the other hand, the morphology of cultured fibroblasts in 3D soft collagen matrices varies from dendritic to bipolar depending on the cell-matrix tension $(2,3)$.

Cell migration is also differentially regulated in a $2 \mathrm{D}$ vs. 3D environment (4). In a stiff 2D surface, cell-surface interaction through the integrin receptor induces massive rearrangement of cytoskeleton proteins. The small GTPases Rac and Rho are involved in the formation of lamellipodia and actin stress fibers, which regulate the attachment of the leading edge and contraction/detachment, respectively $(5,6)$. Coordinated regulation by these small GTPases enables cells to migrate. However, for cells in a 3D environment, the cellmatrix interaction is totally different. Cell extensions become entangled with ECM fibrils, resulting in integrin-independent mechanical interactions (7). Moreover, Rac small GTPase activation causes projection of a dendritic network instead of lamellipodia formation, and the activation of $\mathrm{Rho}$ results in withdrawal of the extensions rather than an increase in stress fibers (8). Therefore, cell migration in a 3D environment may require particular regulatory mechanisms (9).

Recently, we found evidence that microtubules are necessary for the formation of dendritic extensions to resist contractile tension in 3D collagen matrices or low tensile acrylamide surfaces, indicating that the microtubule-dependent mechanism in the low-tension state may be critical for cell morphology and motility. In addition, we also showed that blockade of actomyosin activity using myosin IIA or Rho kinase inhibitor did not affect cell migration in 3D collagen matrices (10). Rather, increasing the myosin IIA motor activity with agonist treatment such as LPA profoundly inhibited cell migration in 3D collagen matrices (11). In light of these observations, we speculated that another cellular motor instead of myosin is required for $3 \mathrm{D}$ cell migration.

In the current study, we found that the inhibition of dynein, a microtubule minus-end motor protein, specifically blocks cell migration on both 2D collagen-coated surfaces and 3D collagen matrices when the myosin motor protein activity has been inhibited. Disrupting the functional complex of dynein with dynein intermediate chain (DIC)-specific siRNA also decreased cell migration in 3D collagen matrices, independent of myosin activity. Cell migration with DIC-silenced cells on the soft acrylamide surface showed that cell migration in a low-tension environment is guided in a dynein motordependent manner. All of these results lead us to conclude that dynein function is likely to be an important regulator of cell migration in low-tension conditions such as $3 \mathrm{D}$ environments. 


\section{Materials and methods}

Materials. DMEM, RPMI-1640 powder and Oligofectamine solution were purchased from Invitrogen (Gaithersburg, MD). Newborn calf serum (NCS) and fetal bovine serum (FBS) were purchased from Hyclone (Logan, UT). Platelet-derived growth factor (PDGF) was obtained from Upstate Biotechnology (Lake Placid, NY). Fatty acid-free bovine serum albumin (BSA), Y27632 and erythro-9-(2-hydroxy-3-nonyl) adenine (EHNA) were obtained from Calbiochem (San Diego, CA). Alexa Fluor 488 phalloidin and propidium iodide (PI) were obtained from Molecular Probes (Eugene, OR). RNase (DNase-free) was purchased from Roche (Indianapolis, IN). Fluoromount G was obtained from Southern Biotechnology Associates (Birmingham, AL). An antibody against human dynein intermediate chain (monoclonal) was obtained from Millipore Corp. (Bedford, MA). Type I rat tail collagen $(10.6 \mathrm{mg} / \mathrm{ml})$ was purchased from BD Biosciences (Bedford, MA).

Cell culture. Early passage human foreskin fibroblast BJ cells were cultured in DMEM supplemented with $10 \%$ NCS. MDA-MB-231 human breast cancer cells were cultured in RPMI supplemented with $10 \%$ FBS. Cell culture and experimental incubations were carried out at $37^{\circ} \mathrm{C}$ in a $5 \% \mathrm{CO}_{2}$ incubator.

For experiments with collagen matrices, cells in neutralized solutions of $1 \mathrm{mg} / \mathrm{ml}$ of collagen were placed in 24-well culture plates (SPL) or seeded on top of collagen matrices after polymerization $\left(2 \times 10^{4}\right.$ cells/matrix). Growth factors and inhibitors were added as described in the figure legends.

For experiments with collagen-coated surfaces, harvested cells $\left(2 \times 10^{4}\right)$ were incubated on $12-\mathrm{mm}$ glass coverslips for the times indicated in the figure legend. The coverslips were coated for $30 \mathrm{~min}$ with $50 \mu \mathrm{g} / \mathrm{ml}$ collagen solutions and then washed with PBS (150 mM NaCl, $3 \mathrm{mM} \mathrm{KCl,} 1 \mathrm{mM} \mathrm{KH}_{2} \mathrm{PO}_{4}$, and $6 \mathrm{mM} \mathrm{Na}_{2} \mathrm{HPO}_{4}, \mathrm{pH}$ 7.3). Subsequently, the cultures were incubated with $1 \mathrm{ml}$ of DMEM or RPMI containing $5 \mathrm{mg} / \mathrm{ml}$ BSA and growth factors or inhibitors as indicated in the figure legends.

Polyacrylamide gels of $10 \mathrm{kPa}$ (8\% acrylamide/0.02\% bis) were prepared and bonded to 3-aminopropyltriethoxysilane (Sigma)-treated 12-mm glass coverslips, followed by treatment with $0.5 \mathrm{mg} / \mathrm{ml}$ of $N$-sulfosuccinimidyl-6-(4'-azido2 '-nitrophenylamino) hexanoate (Pierce), and subsequent activation with $365 \mathrm{~nm}$ UV light for $20 \mathrm{~min}$. Activated gels were coated with $50 \mu \mathrm{g} / \mathrm{ml}$ of collagen solution for $1 \mathrm{~h}$ at $37^{\circ} \mathrm{C}$. The gels were then washed three times with PBS and equilibrated in RPMI.

Cell migration assay. To measure 2D cell migration, cells were incubated overnight on collagen-coated coverslips with media containing $0.1 \%$ FBS. The cell cultures were wounded with a pipette tip and then incubated in media containing $5 \mathrm{mg} / \mathrm{ml}$ BSA and agonists as indicated in the figure legend.

To measure $3 \mathrm{D}$ cell migration, an assay was performed in a modified Boyden chamber with separate lower and upper culture chambers. Cells in neutralized solutions of $1 \mathrm{mg} / \mathrm{ml}$ of collagen were placed on the inner chamber to polymerize for $1 \mathrm{~h}$. One percent FBS/RPMI and 10\% FBS/RPMI were added to the upper and lower chambers, respectively. Both chambers were treated with inhibitors. After $72 \mathrm{~h}$, non-migrated cells on the upper surface were removed with a cotton swab, and those on the lower surface of the filter were fixed and stained with hematoxylin.

To perform the $3 \mathrm{D}$ cell clumping assay, cells were cultured for an initial period of $6 \mathrm{~h}$ on $1 \mathrm{mg} / \mathrm{ml}$ collagen matrices in DMEM containing 10\% FBS. Subsequently, the samples were washed and replaced with $50 \mathrm{ng} / \mathrm{ml}$ PDGF and $10 \% \mathrm{FBS}$ media for an additional $18 \mathrm{~h}$. At the end of the incubation, samples were fixed and stained with phalloidin and PI to visualize actin and nuclei, respectively.

Dynein silencing by siRNA. To knock down dynein, the designed primer pairs for dynein intermediate chain (DIC) siRNA were obtained from GenePharma (Shanghai, China).

siRNA silencing of gene expression in the cells was accomplished as previously described, with minor modifications (12). Mock-transfected cells were treated with the transfection reagent containing mock siRNA.

Immunoblotting. Cells were washed with PBS and lysed with 1X SDS-PAGE sample buffer (50 mM Tris-Cl, $\mathrm{pH} 6.8,2 \%$ SDS, $10 \%$ glycerol, $1 \mathrm{mM}$ DTT) containing protease inhibitors. The lysates were subjected to SDS-PAGE, followed by transfer to a PVDF membrane (Millipore). Transfers were blocked with 5\% skim milk in PBS containing 0.1\% Tween-20 (1X PBST) for $1 \mathrm{~h}$ at room temperature, then incubated with primary antibodies in $3 \% \mathrm{BSA}$ in $1 \mathrm{X}$ PBST for $12 \mathrm{~h}$ at $4{ }^{\circ} \mathrm{C}$; washed three times for $10 \mathrm{~min}$ with $1 \mathrm{X}$ PBST; incubated with secondary antibodies in 5\% skim milk in 1X PBST for $1 \mathrm{~h}$ at room temperature; and washed three times for 10 min with $1 \mathrm{X}$ PBST, after which the signal was developed with ECL reagent.

Fluorescence microscopy. Immunostaining was carried out as described previously (10). For actin staining, we used Alexa Fluor 488-conjugated phalloidin. For PI staining, samples were fixed and stained for $30 \mathrm{~min}$ at room temperature with $8 \mu \mathrm{g} / \mathrm{ml} \mathrm{PI}$ and $20 \mu \mathrm{g} / \mathrm{ml}$ R Nase. Images were collected using a fluorescent microscope (Eclipse 80i, Nikon) using Plan Fluor 10x/0.30, Plan Apo 20x/0.75 and Plan Fluor 40x/0.75 infinitycorrected objectives. Images were acquired using a digital camera (digital sight DS-Qi1Mc, Nikon) and NIS elements image analysis (Nikon). Image processing was carried out using Photoshop 11.0 (Adobe).

\section{Results and Discussion}

The effects of Rho kinase and myosin inhibitors on cell migration. We have previously reported that the cell-specific promigratory and procontractile growth factor environments control cell motility and morphogenesis in different manners (10). That is, PDGF as a promigratory factor induces membrane ruffle and robust cell migration, while LPA or serum as a procontractile factor enhances Rho kinase/myosin signaling and prevents cell migration in $2 \mathrm{D}$ and $3 \mathrm{D}$ environments. Therefore, we speculated that Rho kinase/myosin IIA activity induced by a procontractile factor may inhibit cell migration.

To address this hypothesis, we carried out initial experiments to examine the overall cell morphology via fluorescence imaging of actin using Y27632 to interfere with the Rho 
A
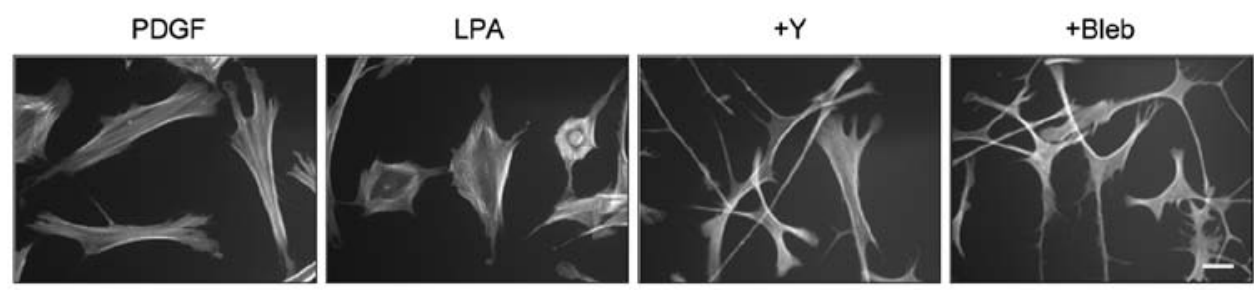

B

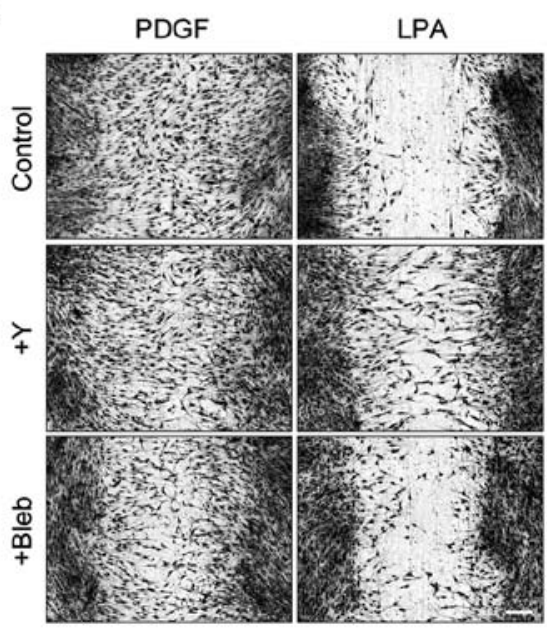

C

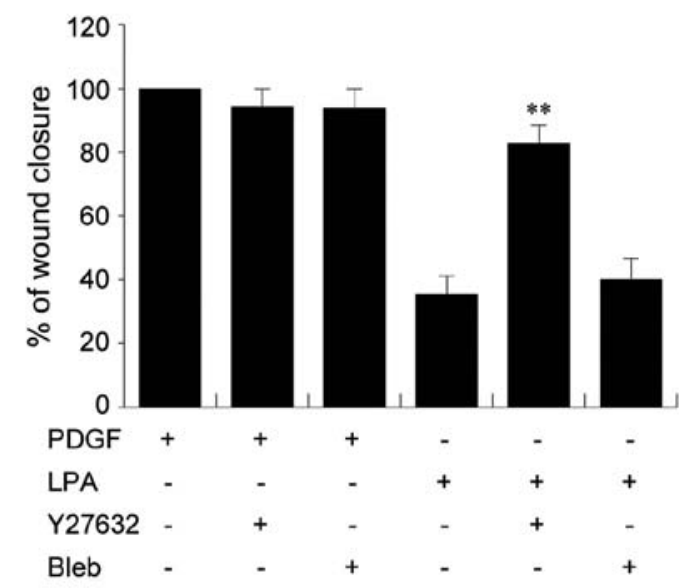

Figure 1. Effects of myosin II and Rho kinase inhibitors on cell migration. (A) BJ cells were incubated for $4 \mathrm{~h}$ on collagen-coated glass coverslips in DMEM

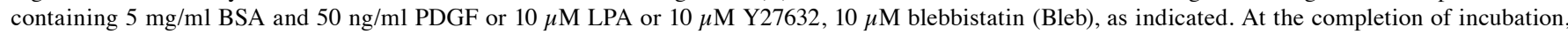
samples were stained for actin. Treatment with Y27632 and blebbistatin altered the cell shape to a wavy and elongated morphology, as observed with PDGF treatment. Scale bar, $50 \mu \mathrm{m}$. (B) BJ cells were serum-starved for $24 \mathrm{~h}$, and a migration assay was performed as described in Materials and methods. Under the LPA condition, treatment with Y27632, but not blebbistatin, enhanced cell migration. Scale bar, $200 \mu \mathrm{m}$. (C) The average wound closure was measured as a percentage of original wound width. ${ }^{* *} \mathrm{P}<0.01$, Student's t-test.

kinase activity and blebbistatin to interfere with the myosin II activity. Inhibition with Rho kinase (+Y) or myosin II (+Bleb) altered cell shape; cells possessed long tails and were wavy but had no actin stress fiber formation, which is nearly similar to the morphology of cells that have been stimulated by PDGF, a well known promigratory factor (11) (Fig. 1A). This result indicates that inhibition of actomyosin activity likely alters the cell's morphology to be more susceptible to cell migration.

The next experiments were carried out to examine whether inhibition of Rho kinase and myosin IIA can restore cell migration upon procontractile growth factor stimulation. Fig. 1B and $\mathrm{C}$ show representative images and quantification of scrape wound closure, respectively. Consistent with results from a previous report, gap closure under PDGF conditions was completely achieved by fibroblast migration within $24 \mathrm{~h}$ (11). In addition, neither Rho kinase nor myosin inhibitors prevented PDGF-induced cell migration, indicating that cell migration in a promigratory environment does not require the Rho kinase/myosin IIA signaling pathway. In contrast, LPA, a typical procontractile factor, failed to induce cell migration, while the Rho kinase inhibitor, Y27632, completely restored the LPA-mediated cell migration. The myosin inhibitor, blebbistatin, also partially recovered the cell migration induced by LPA (Fig. 1B and C).

Effect of the dynein inhibitor on myosin-independent cell migration. Previous experiments have indicated that the myosin motor may play an inhibitory role in cell migration in procontractile growth factor environments. This observation raises the possibility that cells may require substitute motor proteins to generate the driving force for cell migration in the absence of myosin motor activity.

It has been known that dynein, a minus-end microtubule motor, generates a driving force for flagella movement of prokaryote and chromosome segregation during mitosis. In addition, dynein is also known to be a critical regulator of cancer motility and metastasis $(13,14)$. Therefore, we examined the possibility that dynein can control cell migration when myosin activity is absent using a pharmacological inhibitor of dynein, EHNA, which is known to inhibit dynein-microtubule binding by suppression of the ATPase activity of dynein (15).

Since dynein function has been shown to be closely associated with microtubule-organizing center (MTOC) maintenance and re-orientation in response to agonist stimulation (16), we performed an experiment as a control to determine whether MTOC could be modified by EHNA. The MTOC of cells treated with EHNA disappeared in comparison to that in mock-treated cells (Fig. 2A). Thus, we confirmed that EHNA can specifically modify dynein activity, which consequently results in abnormal regulation of MTOC formation.

Next, experiments were carried out to determine whether blockade of dynein activity by EHNA prevents cell migration when actomyosin activity has been blocked. Rho kinase or the dynein inhibitor alone did not inhibit gap closure of in vitro wound healing. However, treatment with a combination of those inhibitors resulted in a profound decrease in wound closure 
A

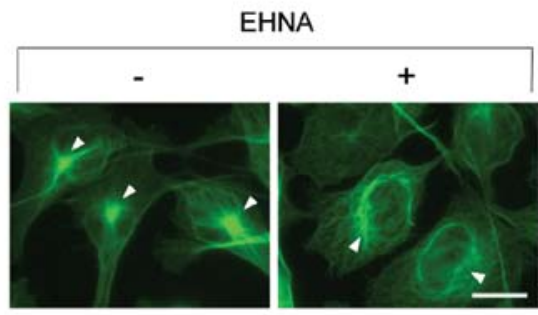

C

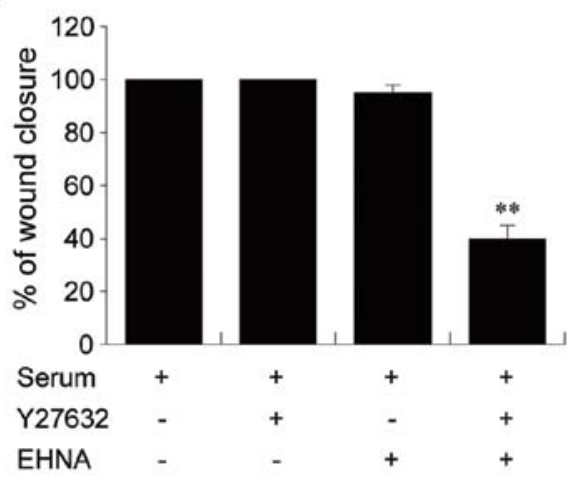

B
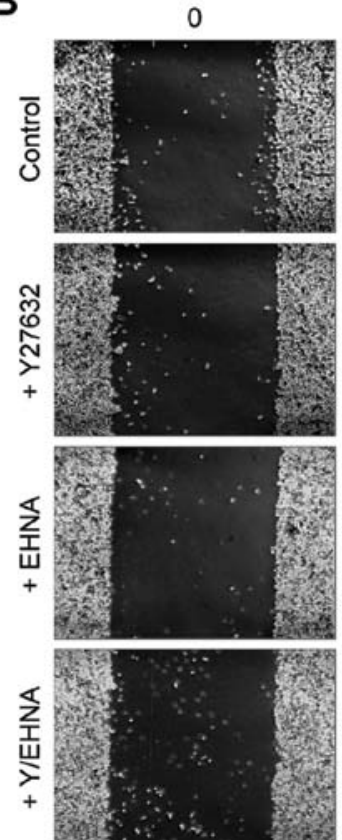

$48(\mathrm{~h})$
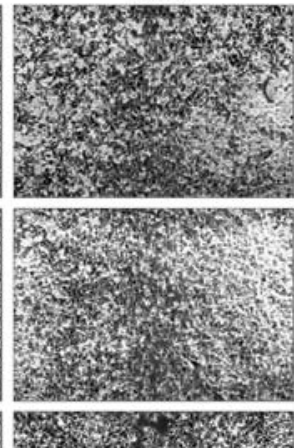
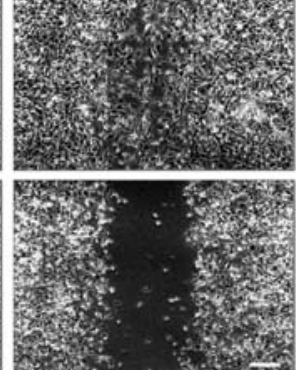

Figure 2. Effects of the dynein inhibitor on Rho kinase-independent cell migration. (A) MDA-MB-231 cells were cultured for $24 \mathrm{~h}$ on collagen-coated coverslips with $500 \mu \mathrm{M}$ of EHNA in RPMI containing 10\% FBS. Samples were stained for $\beta$-tubulin to visualize the microtubule-organizing center (MTOC). EHNA impaired dynein activity, which was assessed based on the disruption of radial microtubule organization. Arrowheads indicate the MTOC. Scale bar, $20 \mu \mathrm{m}$. (B) The migration assay was carried out as described in Materials and methods. Cell migration was inhibited when both inhibitors were added. Scale bar, $200 \mu \mathrm{m}$. (C) Average wound closure was measured as a percentage of original wound width. ${ }^{* *} \mathrm{P}<0.01$, Student's t-test.

A
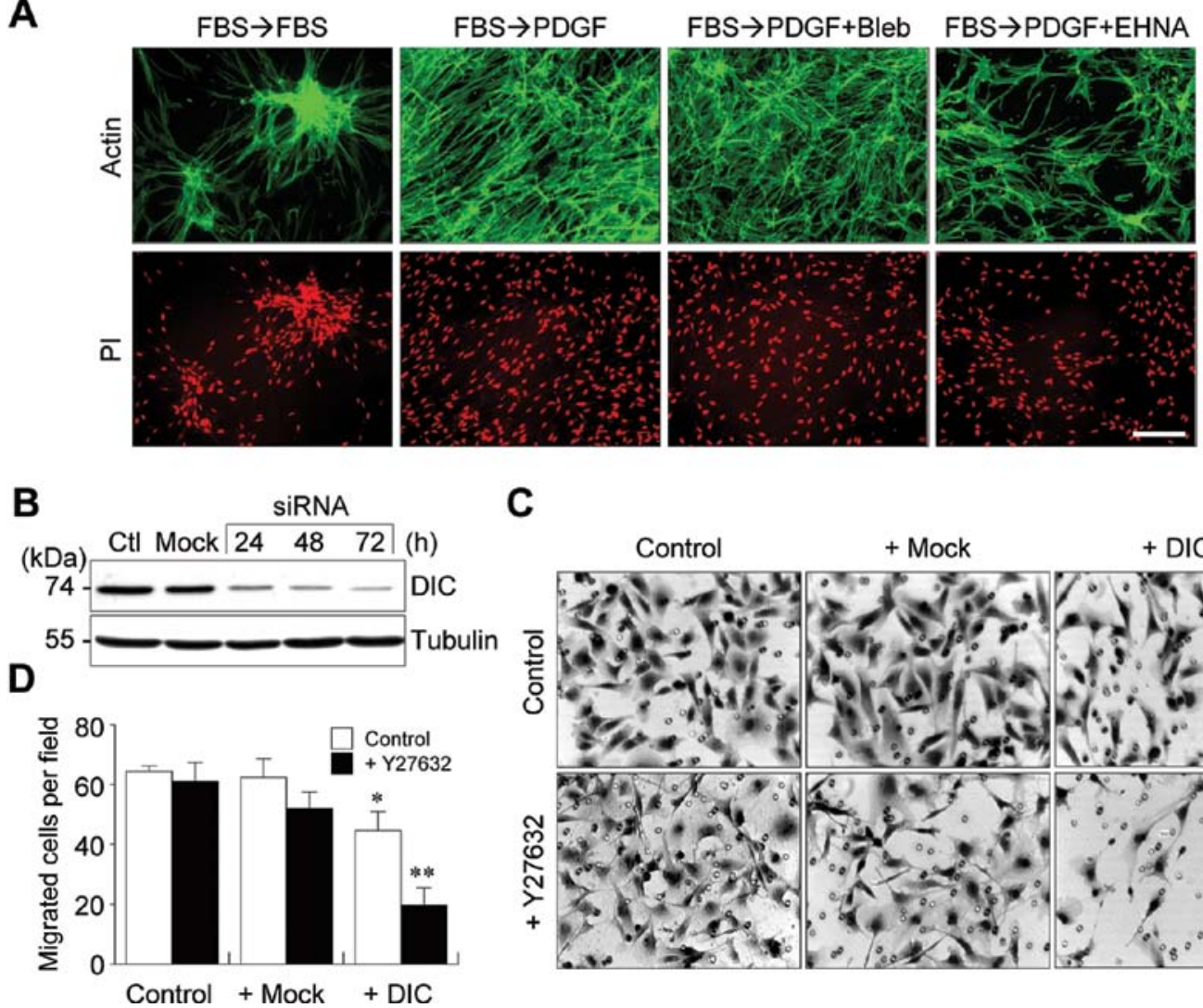

C

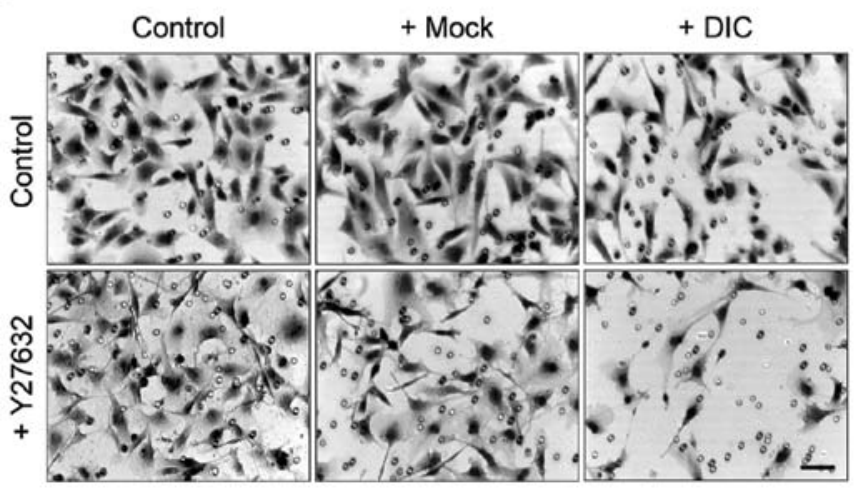

Figure 3. Inhibition of dynein impairs cell migration in a 3D environment. (A) Cell migration patterns on 3D collagen matrices are reversible when FBS and PDGF are present. Blebbistatin (Bleb) did not change the patterns, whereas EHNA markedly inhibited dispersion of cell clusters. Scale bar, $100 \mu \mathrm{m}$. (B) Cells were transfected for $24 \mathrm{~h}$ with DIC-specific siRNA and cultured an additional $24 \mathrm{~h}$ in growth medium without siRNA. Western blotting showed that the expression level of dynein was reduced by siRNA. (C) Transwell assay was performed as described in Materials and methods to observe 3D cell migration. Migration of cells with DIC silencing was markedly inhibited in 3D collagen matrices. Scale bar, $50 \mu \mathrm{m}$. (D) Migrated cells were quantified by counting the cells located in the lower filter. Five random fields were counted. ${ }^{*} \mathrm{P}<0.05 ;{ }^{* *} \mathrm{P}<0.01$, Student's t-test. 
A

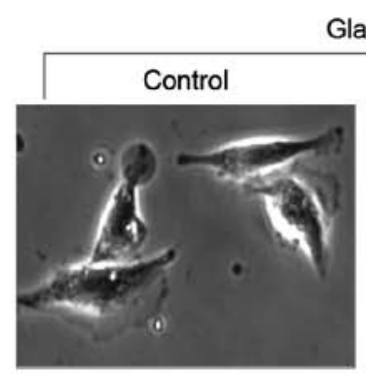

Glass

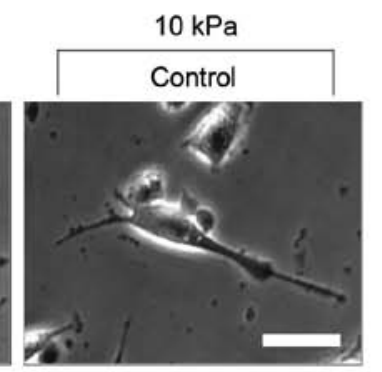

B
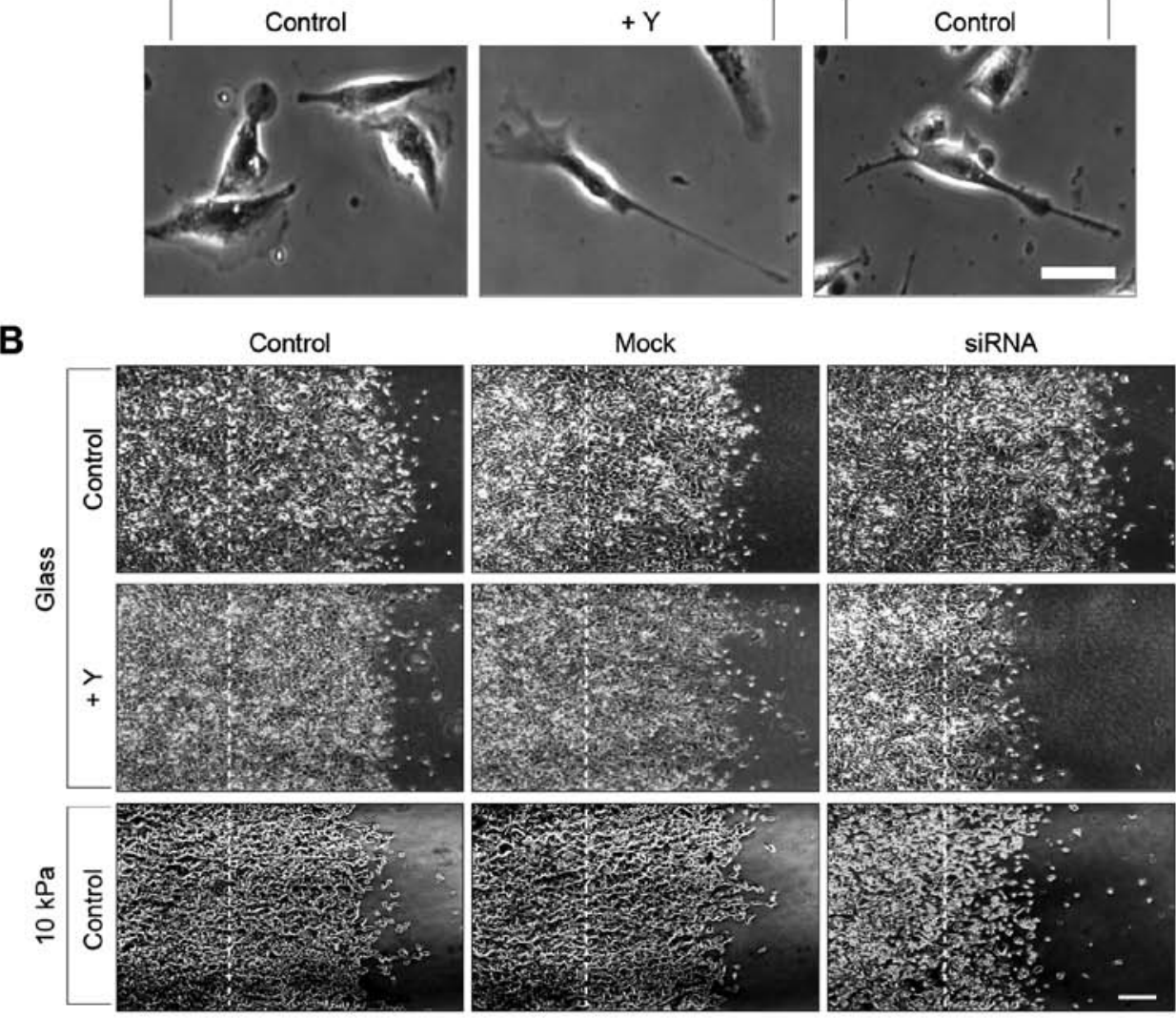

C
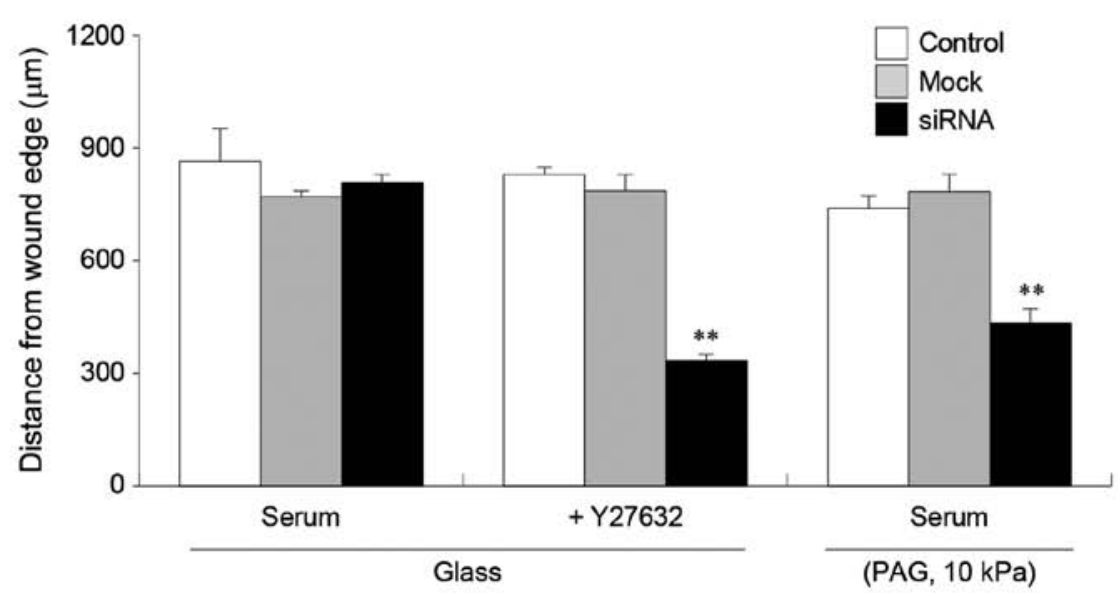

Figure 4. Requirement of dynein function in cell migration on a soft acrylamide gel. Dynein-silenced, mock- and non-transfected (control) cells were harvested and cultured overnight on collagen-coated coverslips. After scrape wounding, the samples were incubated in RPMI containing 10\% FBS for $48 \mathrm{~h}$. (A) Phase contrast images of cell morphology under the same conditions as in (B). Cells on the soft acrylamide had long tails and lamellipodia, which is similar to the morphology observed after treatment with Y27632 on glass coverslips. Scale bar, $50 \mu \mathrm{m}$. (B) Ablation of dynein expression by siRNA resulted in significant decrease in cell migration on a polyacrylamide gel (PAG, $10 \mathrm{kPa}$ ) but not on a 2D stiff glass coverslip. Scale bar, $200 \mu \mathrm{m}$. (C) Migration was quantified by determining the average distance of cell migration from the wound edge. ${ }^{* *} \mathrm{P}<0.01$, Student's t-test

by $\sim 60 \%$ compared to that of control cells (Fig. 2B and C), indicating that dynein motor activity likely accounts for the myosin-independent cell migration.

Effect of dynein inhibitor on cell migration in $3 D$ environments. Recent reports have shown that the requirement for actomyosin contractility in cell migration under 3D matrices is low or absent, even though it is critical in haptokinetic migration in 2D surfaces (17). Our previous research revealed that fibroblast migration in 3D matrices in response to serum caused cell clustering that is completely reversible upon switching to PDGF medium (10). Thus, we took advantage of a system in which cell clusters in serum-containing medium dispersed when using PDGF medium. We aimed to test whether cell dispersion by PDGF stimulation can be inhibited by a dynein inhibitor. Fig. 3A shows that fibroblasts in preformed clusters completely disperse in PDGF medium (FBS $\rightarrow$ PDGF), and the PDGF-induced cell dispersion is not inhibited by blocking the myosin activity with blebbistatin treatment (FBS $\rightarrow$ PDGF+Bleb). Rather, inhibition of myosin activity with 
blebbistatin alone without PDGF induced partial dispersion of the cell clusters (data not shown). However, treatment with the dynein inhibitor prior to adding PDGF profoundly inhibited cell dispersion from the cluster, resulting in the persistence of clusters containing 30-50 cells (FBS $\rightarrow$ PDGF+EHNA). These results support the idea that actomyosin activity is not required for cell migration in 3D matrices, but dynein is likely important for cell migration in a 3D environment where actomyosin activity is absent.

To precisely quantify how many cells can infiltrate the $3 \mathrm{D}$ matrices, 3D collagen matrices containing breast carcinoma cells, MDA-MB-231, were prepared on top of a Transwell chamber, and the number of cells on the bottom of the filter was counted to represent the number of cells moving through the $3 \mathrm{D}$ space.

We used siRNA technology to silence dynein intermediate chain (DIC) expression in order to disrupt the dynein complex in the cells. Fig. 3B shows an example of results obtained from immunoblotting experiments with cell lysates prepared at various time points after DIC-specific siRNA transfection. The level of DIC in DIC siRNA transfected cells, but not mocksiRNA transfected cells, was reduced by $\sim 90 \%$ and remained low for up to $72 \mathrm{~h}$. Fig. 3C and D show representative images of cells on the bottom of the filter and their quantification, respectively. DIC silencing led to profoundly decreased infiltration of cells in 3D collagen matrices compared to that of mock-treated cells. Although migration of the cells through the 3D collagen matrices was not inhibited by treatment with the Rho kinase inhibitor, which is consistent with previous observations that the Rho kinase inhibitor did not suppress 3D migration of fibroblast (18), the movement of DIC-silenced cells through the $3 \mathrm{D}$ matrices was significantly impaired, independent of the Rho kinase.

It has been shown that actin-network expansion at the leading edge, rather than integrin-dependent myosin IIA activity, may be sufficient to provide the motor for leukocyte migration in a $3 \mathrm{D}$ environment where cells do not exert a strong adhesive force (17). We also showed that inhibition of functional myosin IIA activity by treatment with Rho kinase inhibitor promotes the expansion of the actin network and increases massive lamellipodia formation at the leading edge, resulting in enhanced fibroblast migration in 2D surfaces and 3D matrices. However, inhibition of dynein motor activity by treatment with a pharmacological inhibitor or siRNA profoundly impaired the myosin II-independent cell migration in both 2D and 3D milieu. Notably, DIC silencing profoundly decreased cell migration in 3D matrices but not on 2D surfaces, even though myosin IIA activity was maintained, suggesting that the dynein function may be involved in the generation of driving force for cell migration, without resistance from strong adhesive forces such that exist in a 3D environment $(4,19)$. The details of the molecular mechanism underlying dyneindependent cell migration without myosin IIA activity need to be further elucidated.

Effect of dynein inhibition on cell migration in low-tension soft acrylamide matrix. Previous results have indicated that dynein function is likely involved in cell migration under lowtension conditions such as those in 3D collagen matrices. Thus, we performed wound healing experiments with collagen- coated, soft polyacrylamide gels, in which the tension state of the cell-matrix interaction is reduced (3). Collagen-coated acrylamide gels were prepared with ratios of acrylamide and bis-acrylamide previously shown to result in matrix stiffness of $\sim 10 \mathrm{kPa}$, as measured by atomic force microscopy (20). The morphological features of the cells on the $10 \mathrm{kPa}$ acrylamide gel showed that the cell has a long tail with lamellipodia at the leading edge, which is similar to the morphology of cells treated with a Rho kinase inhibitor on a glass surface. This result indicates that cells on a soft acrylamide surface have low-tension cell-matrix interactions (Fig. 4A).

Fig. 4B and $\mathrm{C}$ show the comparison of cell migration with DIC-silenced cells on collagen-coated glass coverslips vs. acrylamide gels. Control and mock siRNA-treated cells have similar extents of migration over $24 \mathrm{~h}$ under both conditions. However, the migration of cells on soft collagen-coated acrylamide matrices is significantly decreased by DIC silencing. Consistent with previous results, migration of cells with DIC silencing is specifically inhibited in the presence of the Rho kinase inhibitor on collagen-coated glass coverslips. Therefore, we conclude that dynein has a major role in cell migration in low-tension substrates such as 3D collagen matrices.

It has been proposed that cell migration on a $2 \mathrm{D}$ surface is controlled by three processes including protrusion of leading, contraction of the cell body and detachment of the tailing edge. In particular, the tractional force exerted by actomyosin activity is critical for cell body contraction, leading to rearward traction of focal contact and forward movement of the cell in the 2D planar surface $(20,21)$. In a 3D environment, however, actomyosin activity of the fibroblast stimulated by serum causes cell clumping instead of dispersion (10). We also recently demonstrated that microtubules play a major role in cell morphology and signaling in low-tension cell-matrix interactions in 3D collagen matrices (3). These results and our findings imply that cells may utilize dynein instead of myosin as a motor for generating a driving force for cell migration under conditions where the cell exerts a low-tension cellmatrix interaction, such as in $3 \mathrm{D}$ matrices.

In contrast to results obtained with Rho kinase inhibitor, direct inhibition of myosin activity by blebbistatin treatment did not increase cell migration upon LPA stimulation. However, we could not rule out the requirement of myosin activity in the low-tension cell-matrix interaction. Since interfering with dynein alone did not affect cell migration on a stiff 2D surface, it can serve as a comprehensive model for the role of motor proteins in cell migration under different mechanical environments.

In conclusion, our research demonstrates that blockade of dynein function inhibits myosin-independent cell migration on 2D surfaces and in 3D matrices. In particular, morphological analysis and wound healing assays with soft acrylamide gels clearly show that dynein function is indispensible for cell migration in a low-tension environment. The molecular dissection of the regulatory proteins involved in dynein-mediated cell migration in the $3 \mathrm{D}$ environment remains to be further investigated.

\section{Acknowledgements}

This study was supported by Chung-Ang University Research Scholarship Grants in 2009. 


\section{References}

1. Brown AE and Discher DE: Conformational changes and signaling in cell and matrix physics. Curr Biol 19: R781-R789, 2009.

2. Rhee S and Grinnell F: Fibroblast mechanics in 3D collagen matrices. Adv Drug Deliv Rev 59: 1299-1305, 2007.

3. Rhee S, Jiang $\mathrm{H}$, Ho CH and Grinnell F: Microtubule function in fibroblast spreading is modulated according to the tension state of cell-matrix interactions. Proc Natl Acad Sci USA 104: 5425-5430, 2007.

4. Zaman MH, Trapani LM, Sieminski AL, Mackellar D, Gong H, Kamm RD, Wells A, Lauffenburger DA and Matsudaira P: Migration of tumor cells in 3D matrices is governed by matrix stiffness along with cell-matrix adhesion and proteolysis. Proc Natl Acad Sci USA 103: 10889-10894, 2006.

5. Ridley AJ and Hall A: The small GTP-binding protein rho regulates the assembly of focal adhesions and actin stress fibers in response to growth factors. Cell 70: 389-399, 1992.

6. Ridley AJ and Hall A: Distinct patterns of actin organization regulated by the small GTP-binding proteins Rac and Rho. Cold Spring Harb Symp Quant Biol 57: 661-671, 1992.

7. Jiang $\mathrm{H}$ and Grinnell F: Cell-matrix entanglement and mechanical anchorage of fibroblasts in three-dimensional collagen matrices. Mol Biol Cell 16: 5070-5076, 2005.

8. Grinnell F: Fibroblast biology in three-dimensional collagen matrices. Trends Cell Biol 13: 264-269, 2003.

9. Friedl P and Brocker EB: The biology of cell locomotion within three-dimensional extracellular matrix. Cell Mol Life Sci 57: 41-64, 2000.

10. Rhee S, Ho CH and Grinnell F: Promigratory and procontractile growth factor environments differentially regulate cell morphogenesis. Exp Cell Res 316: 232-244, 2010.

11. Jiang $\mathrm{H}$, Rhee S, Ho CH and Grinnell F: Distinguishing fibroblast promigratory and procontractile growth factor environments in 3-D collagen matrices. FASEB J 22: 2151-2160, 2008.
12. Rhee S and Grinnell F: P21-activated kinase 1: convergence point in PDGF- and LPA-stimulated collagen matrix contraction by human fibroblasts. J Cell Biol 172: 423-432, 2006.

13. Grinberg-Rashi H, Ofek E, Perelman M, Skarda J, Yaron P, Hajduch M, Jacob-Hirsch J, Amariglio N, Krupsky M, Simansky DA, et al: The expression of three genes in primary non-small cell lung cancer is associated with metastatic spread to the brain. Clin Cancer Res 15: 1755-1761, 2009.

14. Ishikawa K, Kamohara Y, Tanaka F, Haraguchi N, Mimori K, Inoue $\mathrm{H}$ and Mori $\mathrm{M}$ : Mitotic centromere-associated kinesin is a novel marker for prognosis and lymph node metastasis in colorectal cancer. Br J Cancer 98: 1824-1829, 2008.

15. Bouchard P, Penningroth SM, Cheung A, Gagnon C and Bardin CW: erythro-9-[3-(2-Hydroxynonyl)]adenine is an inhibitor of sperm motility that blocks dynein ATPase and protein carboxylmethylase activities. Proc Natl Acad Sci USA 78: 1033-1036, 1981.

16. Gomes ER, Jani S and Gundersen GG: Nuclear movement regulated by Cdc42, MRCK, myosin, and actin flow establishes MTOC polarization in migrating cells. Cell 121: 451-463, 2005.

17. Lammermann T, Bader BL, Monkley SJ, Worbs T, WedlichSoldner R, Hirsch K, Keller M, Forster R, Critchley DR, Fassler R, et al: Rapid leukocyte migration by integrin-independent flowing and squeezing. Nature 453: 51-55, 2008.

18. Nakayama M, Amano M, Katsumi A, Kaneko T, Kawabata S, Takefuji M and Kaibuchi K: Rho-kinase and myosin II activities are required for cell type and environment specific migration. Genes Cells 10: 107-117, 2005.

19. Pedersen JA and Swartz MA: Mechanobiology in the third dimension. Ann Biomed Eng 33: 1469-1490, 2005

20. Lo CM, Wang HB, Dembo M and Wang YL: Cell movement is guided by the rigidity of the substrate. Biophys J 79: 144-152, 2000.

21. Li S, Guan JL and Chien S: Biochemistry and biomechanics of cell motility. Annu Rev Biomed Eng 7: 105-150, 2005. 